\title{
On the optimization of the principal eigenvalue for single-centre point-interaction operators in a bounded region
}

\author{
Pavel Exner*, Andrea Mantile ${ }^{\dagger}$
}

\begin{abstract}
We investigate relations between spectral properties of a single-centre point-interaction Hamiltonian describing a particle confined to a bounded domain $\Omega \subset \mathbb{R}^{d}, d=2,3$, with Dirichlet boundary, and the geometry of $\Omega$. For this class of operators Krein's formula yields an explicit representation of the resolvent in terms of the integral kernel of the unperturbed one, $\left(-\Delta_{\Omega}^{D}+z\right)^{-1}$. We use a moving plane analysis to characterize the behaviour of the ground-state energy of the Hamiltonian with respect to the point-interaction position and the shape of $\Omega$, in particular, we establish some conditions showing how to place the interaction to optimize the principal eigenvalue.
\end{abstract}

\section{Introduction}

Relations between geometry of a domain and spectral properties of corresponding operators belong to the most traditional question in mathematical physics; one can recall, e.g., the FaberKrahn inequality [Fa23, Kr25] or the Payne-Pólya-Weinberger conjecture [PPW55] proved by Ashbaugh and Benguria AB92a, AB92b. A more recent example concerns the situation where the domain in question is not simply connected and one asks, in particular, how to place a circular hard-wall obstacle within a circular planar cavity to minimize the ground-state eigenvalue; it appears that the minimum is reached when the obstacle touches the boundary HKK01.

In connection with the last mentioned problem one can also ask what happens if such a "hard" obstacle is replaced by another object, say, by a potential barrier or well. In this paper we are going to address this question in the particular case when such a potential is singular, in other words, a point interaction. Recalling basic results about these interactions AGHH05 we see that the problem makes sense in dimension $d \leq 3$. Furthermore, a simple perturbative argument shows that in the one dimensional situation the answer may depend on the sign of the $\delta$ potential; we restrict here our attention to the more singular case of dimension $d=2,3$.

Problems of this type were to our knowledge solved so far only in cases where the domain has a simple geometry such as a straight strip in $\mathbb{R}^{2}$, see EGŠT96, or a planar layer in $\mathbb{R}^{3}$, see EN02, where the eigenvalue problem can be solved more or less explicitly. Here we consider that the domain $\Omega$, to which the particle is confined, is bounded and otherwise quite general, see below. The operator of interest will be the corresponding Dirichlet Laplacian perturbed by a single point interaction of a fixed coupling constant; we will ask about the dependence of its principal eigenvalue on the perturbation position. Using a figurative expression, to be made

\footnotetext{
*Department of Theoretical Physics, NPI, Academy of Sciences, 25068 Řež near Prague, and Doppler Institute, Czech Technical University, Břehová 7, 11519 Prague, Czechia.

†Doppler Institute, Czech Technical University, Břehová 7, 11519 Prague, Czechia, and IRMAR, Université Rennes 1, Campus de Beaulieu, 35042, Rennes Cedex, France.
} 
precise below, we are going to show that the ground-state energy increases as the point moves towards the boundary of $\Omega$, in contrast to the case of a Dirichlet obstacle mentioned above.

Our method is based on the fact that one is able to express the resolvent of the operator in question by means of the Krein's formula. The resulting spectral condition allow us to characterize the principal eigenvalue as a function of the interaction position in a non perturbative setting. Then we exploit the maximum principle and a domain reflection technique, analogous to the one used in [HKK01, to demonstrate our main result, Theorem 4.3] expressing strict monotonicity of the principal eigenvalue with respect to certain directions. Using this conclusion, we formulate then some conditions under which the eigenvalue reaches its minimum value for a fixed $\Omega$.

\section{Confined point interactions in two and three dimensions}

The definition domain and the spectral properties of point interaction Hamiltonians in dimensions two and three are usually expressed in terms of the free Green function, i.e. the integral kernel of the operator $(-\Delta+z)^{-1}$, cf. AGHH05. This is true both if the configuration space is the whole $\mathbb{R}^{d}$ or if the particle is confined to a subset $\Omega$ of it by a hard wall corresponding to Dirichlet boundary condition. In the latter case Green's function is defined by the equation

$$
\left\{\begin{array}{l}
(-\Delta+z) \mathcal{G}_{0}^{z}\left(\underline{x}, \underline{x}^{\prime}\right)=\delta\left(\underline{x}-\underline{x}^{\prime}\right) \\
\left.\mathcal{G}_{0}^{z}\left(\underline{x}, \underline{x}^{\prime}\right)\right|_{\underline{x} \in \partial \Omega}=0
\end{array} ; \quad \underline{x}^{\prime} \in \Omega\right.
$$

which admits a solution in $L^{2}(\Omega)$ whenever $-z$ does not belong to the spectrum of the Dirichlet Laplacian $-\Delta_{\Omega}^{D}$ defined in the standard way through the associated quadratic form RS78. Throughout this paper, $\Omega$ is supposed to be an open regular set in $\mathbb{R}^{d}, d=2,3$, bounded and connected, not necessarily simply; we assume that $\partial \Omega$ is piecewise $C^{1}$.

Under these hypotheses $-\Delta_{\Omega}^{D}$ has a purely discrete spectrum; we denote by $\left\{\lambda_{n}\right\}_{n \in \mathbb{N}_{0}}$, where $\mathbb{N}_{0}:=\mathbb{N} \cup\{0\}$, its eigenvalues, and by $\left\{\psi_{n, k}\right\}_{\substack{k=1 \\ n \in \mathbb{N}_{0}}}^{N_{n}}$ the corresponding system of eigenfunctions, $N_{n}$ being the multiplicity of the $n$-th eigenvalue. Projecting (2.1) on the vectors $\psi_{n, k}$, it is easy to check the validity of the following standard Fourier expansion of $\mathcal{G}_{0}^{z}$,

$$
\mathcal{G}_{0}^{z}\left(\underline{x}, \underline{x}^{\prime}\right)=\sum_{\substack{n \in \mathbb{N}_{0} \\ k \leq N_{n}}} \frac{\psi_{n, k}\left(\underline{x}^{\prime}\right) \psi_{n, k}(\underline{x})}{\lambda_{n}+z} .
$$

An alternative representation of the kernel, which will be extensively used in this paper, can be given in terms of the 'free' Green's function $\mathcal{G}^{z}\left(\underline{x}, \underline{x}^{\prime}\right)$, in other words, the integral kernel of $(-\Delta+z)^{-1}$ in the whole spact 1 ,

$$
\begin{aligned}
& \mathcal{G}^{z}\left(\underline{x}, \underline{x}^{\prime}\right)=\frac{1}{2 \pi} K_{0}\left(\sqrt{z}\left|\underline{x}-\underline{x}^{\prime}\right|\right) \quad \text { in } \mathbb{R}^{2} \\
& \mathcal{G}^{z}\left(\underline{x}, \underline{x}^{\prime}\right)=\frac{e^{-\sqrt{z}\left|\underline{x}-\underline{x}^{\prime}\right|}}{4 \pi\left|\underline{x}-\underline{x}^{\prime}\right|} \quad \text { in } \mathbb{R}^{3}
\end{aligned}
$$

Here $K_{0}$ denotes the Macdonald (or modified Hankel) function. Using the boundary conditions in (2.1), we can express the kernel of interest as

$$
\mathcal{G}_{0}^{z}\left(\underline{x}, \underline{x}^{\prime}\right)=\mathcal{G}^{z}\left(\underline{x}, \underline{x}^{\prime}\right)-h\left(\underline{x}, \underline{x}^{\prime}, \sqrt{z}\right)
$$

\footnotetext{
${ }^{1}$ Here and in the following we use the convention in which the negative real axis represents the cut of the square root $\sqrt{z}$ in the complex plane.
} 
where $\mathcal{G}^{z}$ is defined by $\left(\underline{2.3)}-(2.4)\right.$ and $h\left(\underline{x}, \underline{x}^{\prime}, \sqrt{z}\right)$ solves the boundary value problem

$$
\left\{\begin{array}{l}
(-\Delta+z) h\left(\underline{x}, \underline{x}^{\prime}, \sqrt{z}\right)=0 \\
\left.h\left(\underline{x}, \underline{x}^{\prime}, \sqrt{z}\right)\right|_{\underline{x} \in \partial \Omega}=\left.\mathcal{G}^{z}\left(\underline{x}, \underline{x}^{\prime}\right)\right|_{\underline{x} \in \partial \Omega}
\end{array} \quad \text { for any } \underline{x}^{\prime} \in \Omega\right.
$$

In the next step we construct the operator which will be our main object perturbing $-\Delta_{\Omega}^{D}$ by a single point interaction with the support at a point $\underline{x}_{0} \in \Omega$. Such Hamiltonians are defined by the self-adjoint extensions of the symmetric operator

$$
\left\{\begin{array}{l}
D\left(H_{0}\right)=\left\{\psi \in H^{2} \cap H_{0}^{1}(\Omega) \mid \psi\left(\underline{x}_{0}\right)=0\right\} \\
H_{0} \psi=-\Delta \psi
\end{array}\right.
$$

Following the von Neumann theory [AG63, RS75, we observe that the restriction (2.7) has deficiency indices $(1,1)$; consequently, we arrive at a one-parameter family of self-adjoint operators $H_{\alpha}$. For fixed $\alpha \in \mathbb{R}, \lambda \in \mathbb{C} \backslash \mathbb{R}$ and a domain $\Omega \subset \mathbb{R}^{2}$ we have

$$
\begin{gathered}
D\left(H_{\alpha}\right)=\left\{\psi \in L^{2}(\Omega) \mid \psi=\phi^{\lambda}+q \mathcal{G}_{0}^{\lambda}\left(\cdot, \underline{x}_{0}\right), \phi^{\lambda} \in H^{2} \cap H_{0}^{1}(\Omega),\right. \\
\left.\phi^{\lambda}\left(\underline{x}_{0}\right)=\frac{q}{2 \pi}\left(\alpha-\ln \sqrt{\lambda}-2 \pi h\left(\underline{x}_{0}, \underline{x}_{0}, \sqrt{\lambda}\right)\right)\right\}
\end{gathered}
$$

while for $\Omega \subset \mathbb{R}^{3}$ the operator domain is

$$
\begin{gathered}
D\left(H_{\alpha}\right)=\left\{\psi \in L^{2}(\Omega) \mid: \psi=\phi^{\lambda}+q \mathcal{G}_{0}^{\lambda}\left(\cdot, \underline{x}_{0}\right), \phi^{\lambda} \in H^{2} \cap H_{0}^{1}(\Omega),\right. \\
\left.\phi^{\lambda}\left(\underline{x}_{0}\right)=q\left(\alpha+\frac{\sqrt{\lambda}}{4 \pi}+h\left(\underline{x}_{0}, \underline{x}_{0}, \sqrt{\lambda}\right)\right)\right\}
\end{gathered}
$$

In both cases the parameter $\lambda$ determines a representation of the operator domain, roughly speaking, a split between the regular and singular part; for a fixed choice of $\lambda$ the action of $H_{\alpha}$ is the following

$$
H_{\alpha} \psi=-\Delta \phi^{\lambda}-\lambda q \mathcal{G}_{0}^{\lambda}\left(\cdot, \underline{x}_{0}\right) .
$$

It is convenient to include into this description also infinite values of the parameter $\alpha$, in which case the coefficient $q$ of the singular part - sometimes referred to as the charge of the state vanishes. It is equivalent to the absence of the point interaction: the domain reduces in this case to $H^{2} \cap H_{0}^{1}(\Omega)$ and the self-adjoint extensions corresponding to $\alpha= \pm \infty$ is identified with the unperturbed operator $-\Delta_{\Omega}^{D}$.

Making use of Krein's formula AG63 the action of the resolvent, $R_{z}^{\alpha}=\left(H_{\alpha}+z\right)^{-1}$ on $L^{2}(\Omega)$, can be expressed as a rank-one perturbation of its 'free' counterpart $(-\Delta+z)^{-1}$, specifically

$$
R_{z}^{\alpha} \varphi=(-\Delta+z)^{-1} \varphi+q_{z}\left((-\Delta+z)^{-1} \varphi\right)\left(\underline{x}_{0}\right) \mathcal{G}_{0}^{z}\left(\cdot, \underline{x}_{0}\right) .
$$

In this formula, of course, the first term is the regular part of the function $R_{z}^{\alpha} \varphi \in D\left(H_{\alpha}\right)$ while the value $q_{z}\left((-\Delta+z)^{-1} \varphi\right)\left(\underline{x}_{0}\right)$ denotes the corresponding charge. Using the boundary conditions in (2.8) and (2.9) we can identify the coefficient $q_{z}$ with

$$
\begin{gathered}
q_{z}=\left(\alpha-\ln \sqrt{z}-2 \pi h\left(\underline{x}_{0}, \underline{x}_{0}, \sqrt{z}\right)\right)^{-1}, \quad \Omega \subset \mathbb{R}^{2} \\
q_{z}=\left(\alpha+\frac{\sqrt{z}}{4 \pi}+h\left(\underline{x}_{0}, \underline{x}_{0}, \sqrt{z}\right)\right)^{-1}, \quad \Omega \subset \mathbb{R}^{3}
\end{gathered}
$$


Furthermore, the Fourier expansion

$$
(-\Delta+z)^{-1} \varphi=\sum_{\substack{n \in \mathbb{N}_{0} \\ k \leq N_{n}}} \frac{\left(\varphi, \psi_{n, k}\right)}{\lambda_{n}+z} \psi_{n, k}
$$

yields the following explicit expression

$$
R_{z}^{\alpha} \varphi=\sum_{\substack{n \in \mathbb{N}_{0} \\ k \leq N_{n}}} \frac{\left(\varphi, \psi_{n, k}\right)}{\lambda_{n}+z} \psi_{n, k}+2 \pi \sum_{\substack{n \in \mathbb{N}_{0} \\ k \leq N_{n}}} \frac{\left(\varphi, \psi_{n, k}\right)}{\lambda_{n}+z} \frac{\psi_{n, k}\left(\underline{x}_{0}\right)}{\alpha-\ln \sqrt{z}-2 \pi h\left(\underline{x}_{0}, \underline{x}_{0}, \sqrt{z}\right)} \mathcal{G}_{0}^{z}\left(\cdot, \underline{x}_{0}\right)
$$

for $\Omega \subset \mathbb{R}^{2}$, and its counterpart

$$
R_{z}^{\alpha} \varphi=\sum_{\substack{n \in \mathbb{N}_{0} \\ k \leq N_{n}}} \frac{\left(\varphi, \psi_{n, k}\right)}{\lambda_{n}+z} \psi_{n, k}+\sum_{\substack{n \in \mathbb{N}_{0} \\ k \leq N_{n}}} \frac{\left(\varphi, \psi_{n, k}\right)}{\lambda_{n}+z} \frac{\psi_{n, k}\left(\underline{x}_{0}\right)}{\alpha+\frac{\sqrt{z}}{4 \pi}+h\left(\underline{x}_{0}, \underline{x}_{0}, \sqrt{z}\right)} \mathcal{G}_{0}^{z}\left(\cdot, \underline{x}_{0}\right)
$$

for $\Omega \subset \mathbb{R}^{3}$.

\section{The principal eigenvalue of $H_{\alpha}$}

As usual, the Krein formula allows us to determine the spectrum through the denominator of the perturbation term. In particular, it follows from the resolvent equations (2.15)-(2.16) that the spectrum of $H_{\alpha}$ is formed by the solutions of the equations

$$
\begin{aligned}
& \alpha-\ln \sqrt{-\xi}-2 \pi h\left(\underline{x}_{0}, \underline{x}_{0}, \sqrt{-\xi}\right)=0, \quad \Omega \subset \mathbb{R}^{2} \\
& \alpha+\frac{\sqrt{-\xi}}{4 \pi}+h\left(\underline{x}_{0}, \underline{x}_{0}, \sqrt{-\xi}\right)=0, \quad \Omega \subset \mathbb{R}^{3}
\end{aligned}
$$

to which one has to add eigenvalues of $-\Delta_{\Omega}^{D}$, the degenerate ones in any case and the nondegenerate ones, $\lambda_{\bar{n}}$, provided that the corresponding eigenfunction $\psi_{\bar{n}}$ satisfies the condition (see e.g. in BFM07])

$$
\psi_{\bar{n}}\left(\underline{x}_{0}\right)=0
$$

This does not concern, however, the bottom of the spectrum which we are interested in here, because the ground state of $-\Delta_{D}^{\Omega}$ is non-degenerate and can be represented by a positive function.

The subject of this section is the principal eigenvalue of the point-interaction operator $H_{\alpha}$. We are going to show, in particular, that for any $\alpha \in \mathbb{R}$ there exists a unique simple eigenvalue of $H_{\alpha}$ below the spectral threshold of $-\Delta_{\Omega}^{D}$. As a preliminary, we need to characterize the derivatives of $h\left(\underline{x}_{0}, \underline{x}_{0}, \sqrt{z}\right)$ w.r.t. the variable $\sqrt{z}$.

Lemma 3.1 Let $z$ be a positive real number; the function $y \mapsto h\left(\underline{x}, \underline{x}^{\prime}, y\right)$, defined by (2.6) with $y:=\sqrt{z}$, satisfies the conditions

$$
\begin{aligned}
& \frac{1}{y}\left(\frac{1}{y}+2 \pi \partial_{y} h\left(\underline{x}^{\prime}, \underline{x}^{\prime}, y\right)\right)>0, \quad \Omega \subset \mathbb{R}^{2} \\
& \frac{1}{y}\left(\frac{1}{4 \pi}+\partial_{y} h\left(\underline{x}^{\prime}, \underline{x}^{\prime}, y\right)\right)>0, \quad \Omega \subset \mathbb{R}^{3}
\end{aligned}
$$

Furthermore, for any $z<0$ and $h\left(\underline{x}, \underline{x}^{\prime}, i y\right)$, defined by (2.6) with $y:=\sqrt{|z|}$, we have

$$
\begin{aligned}
& \frac{\pi}{2}+2 \pi \operatorname{Im} h\left(\underline{x}^{\prime}, \underline{x}^{\prime}, i y\right)=0, \quad \Omega \subset \mathbb{R}^{2} \\
& \frac{y}{4 \pi}+\operatorname{Im} h\left(\underline{x}^{\prime}, \underline{x}^{\prime}, i y\right)=0, \quad \Omega \subset \mathbb{R}^{3}
\end{aligned}
$$


and

$$
\begin{aligned}
& \frac{1}{y}+2 \pi \partial_{y} \operatorname{Re} h\left(\underline{x}^{\prime}, \underline{x}^{\prime}, i y\right)<0, \quad \Omega \subset \mathbb{R}^{2} \\
& \partial_{y} \operatorname{Re} h\left(\underline{x}^{\prime}, \underline{x}^{\prime}, i y\right)<0, \quad \Omega \subset \mathbb{R}^{3}
\end{aligned}
$$

Proof. We start with the 3D case. Let $z_{j}, j=1,2$, be a pair of positive values; setting $y_{j}:=\sqrt{z_{j}}$ we get from equation (2.5) in combination with the first resolvent formula the relation

$$
\left(y_{1}^{2}-y_{2}^{2}\right)\left(\mathcal{G}_{0}^{y_{1}^{2}}, \mathcal{G}_{0}^{y_{2}^{2}}\right)_{L^{2}(\Omega)}=\lim _{\underline{x} \rightarrow \underline{x}^{\prime}}\left[\mathcal{G}^{y_{2}^{2}}\left(\underline{x}, \underline{x}^{\prime}\right)-\mathcal{G}^{y_{1}^{2}}\left(\underline{x}, \underline{x}^{\prime}\right)\right]+h\left(\underline{x}^{\prime}, \underline{x}^{\prime}, y_{1}\right)-h\left(\underline{x}^{\prime}, \underline{x}^{\prime}, y_{2}\right),
$$

where $(\cdot, \cdot)_{L^{2}(\Omega)}$ denotes the scalar product in $L^{2}(\Omega)$. The limit at the r.h.s. is easily seen to be

$$
\lim _{\underline{x \rightarrow \underline{x}^{\prime}}}\left[\mathcal{G}^{y_{2}^{2}}\left(\underline{x}, \underline{x}^{\prime}\right)-\mathcal{G}^{y_{1}^{2}}\left(\underline{x}, \underline{x}^{\prime}\right)\right]=\frac{1}{4 \pi}\left(y_{1}-y_{2}\right)
$$

Substituting this expression into (3.6) we get

$$
\left(y_{1}+y_{2}\right)\left(\mathcal{G}_{0}^{y_{1}^{2}}, \mathcal{G}_{0}^{y_{2}^{2}}\right)_{L^{2}(\Omega)}=\frac{1}{4 \pi}+\frac{h\left(\underline{x}^{\prime}, \underline{x}^{\prime}, y_{1}\right)-h\left(\underline{x}^{\prime}, \underline{x}^{\prime}, y_{2}\right)}{y_{1}-y_{2}},
$$

and consequently, in the limit $y_{1} \rightarrow y_{2}$ we arrive at

$$
\frac{1}{4 \pi}+\partial_{y} h\left(\underline{x}^{\prime}, \underline{x}^{\prime}, y_{2}\right)=2 y_{2}\left\|\mathcal{G}_{0}^{y_{2}^{2}}\right\|_{L^{2}(\Omega)}^{2}>0
$$

On the other hand, for negative values of $z$ a similar argument yields

$$
\left(y_{1}^{2}-y_{2}^{2}\right)\left(\mathcal{G}_{0}^{-y_{1}^{2}}, \mathcal{G}_{0}^{-y_{2}^{2}}\right)_{L^{2}(\Omega)}=\lim _{\underline{x} \rightarrow \underline{x}^{\prime}}\left[\mathcal{G}^{-y_{1}^{2}}\left(\underline{x}, \underline{x}^{\prime}\right)-\left(\mathcal{G}^{-y_{2}^{2}}\right)^{*}\left(\underline{x}, \underline{x}^{\prime}\right)\right]-h\left(\underline{x}^{\prime}, \underline{x}^{\prime}, i y_{1}\right)+h^{*}\left(\underline{x}^{\prime}, \underline{x}^{\prime}, i y_{2}\right)
$$

with the asterisk denoting complex conjugation and $y_{j}:=\sqrt{\left|z_{j}\right|}$. The limit at the r.h.s. is

$$
\lim _{\underline{x} \rightarrow \underline{x}^{\prime}}\left[\mathcal{G}^{-y_{1}^{2}}\left(\underline{x}, \underline{x}^{\prime}\right)-\left(\mathcal{G}^{-y_{2}^{2}}\right)^{*}\left(\underline{x}, \underline{x}^{\prime}\right)\right]=-\frac{i}{4 \pi}\left(y_{1}+y_{2}\right)
$$

from which we get a relation replacing (3.8), namely

$$
\left(y_{1}^{2}-y_{2}^{2}\right)\left(\mathcal{G}_{0}^{-y_{1}^{2}}, \mathcal{G}_{0}^{-y_{2}^{2}}\right)_{L^{2}(\Omega)}=-\frac{i}{4 \pi}\left(y_{1}+y_{2}\right)-h\left(\underline{x}^{\prime}, \underline{x}^{\prime}, i y_{1}\right)+h^{*}\left(\underline{x}^{\prime}, \underline{x}^{\prime}, i y_{2}\right) .
$$

The second one of the relations (3.4) then follows from here by setting $y_{1}=y_{2}$. As for the real part of (3.10) given by

$$
\begin{aligned}
\frac{1}{2}\left(y_{1}^{2}-y_{2}^{2}\right)\left[\left(\mathcal{G}_{0}^{-y_{1}^{2}}, \mathcal{G}_{0}^{-y_{2}^{2}}\right)_{L^{2}(\Omega)}+\left(\mathcal{G}_{0}^{-y_{2}^{2}}, \mathcal{G}_{0}^{-y_{1}^{2}}\right)_{L^{2}(\Omega)}\right]= \\
\quad=\lim _{\underline{x} \rightarrow \underline{x}^{\prime}}\left[\operatorname{Re} \mathcal{G}^{-y_{1}^{2}}\left(\underline{x}, \underline{x}^{\prime}\right)-\operatorname{Re} \mathcal{G}^{-y_{2}^{2}}\left(\underline{x}, \underline{x}^{\prime}\right)\right]-\operatorname{Re} h\left(\underline{x}^{\prime}, \underline{x}^{\prime}, i y_{1}\right)+\operatorname{Re} h\left(\underline{x}^{\prime}, \underline{x}^{\prime}, i y_{2}\right)
\end{aligned}
$$

we notice that the first term at the r.h.s. is in fact zero; dividing the remaining ones by $y_{1}-y_{2}$ and taking the limit as $y_{1} \rightarrow y_{2}$, we arrive at

$$
-\partial_{y} \operatorname{Re} h\left(\underline{x}^{\prime}, \underline{x}^{\prime}, i y_{2}\right)=2 y_{2}\left\|\mathcal{G}_{0}^{-y_{2}^{2}}\right\|_{L^{2}(\Omega)}^{2}>0 .
$$


In the $2 \mathrm{D}$ case the validity of relations (3.3), (3.4) and (3.5) can be checked following the same idea. Taking into account the logarithmic singularity of $\mathcal{G}^{z}\left(\underline{x}, \underline{x}^{\prime}\right)$ as $\underline{x} \rightarrow \underline{x}^{\prime}$ — see, e.g., AS72 — we find for $z>0$

$$
\lim _{\underline{x} \rightarrow \underline{x}^{\prime}}\left[\mathcal{G}^{y_{2}^{2}}\left(\underline{x}, \underline{x}^{\prime}\right)-\mathcal{G}^{y_{1}^{2}}\left(\underline{x}, \underline{x}^{\prime}\right)\right]=\frac{1}{2 \pi}\left(\ln y_{1}-\ln y_{2}\right),
$$

while for $z<0$ we have

$$
\lim _{\underline{x} \rightarrow \underline{x}^{\prime}}\left[\mathcal{G}^{-y_{1}^{2}}\left(\underline{x}, \underline{x}^{\prime}\right)-\left(\mathcal{G}^{-y_{2}^{2}}\right)^{*}\left(\underline{x}, \underline{x}^{\prime}\right)\right]=\frac{1}{2 \pi}\left(-i \pi+\ln y_{1}-\ln y_{2}\right) ;
$$

this concludes the proof.

In the next two lemmata, we deal with the solutions of equations (3.1) below the spectrum of $-\Delta_{\Omega}^{D}$. We are going to show that for a fixed real $\alpha$ there is a unique such solution. It is convenient to treat the $2 \mathrm{D}$ and $3 \mathrm{D}$ cases separately.

Lemma 3.2 Let $\lambda_{0}$ denote the first eigenvalue of $-\Delta_{\Omega}^{D}$ corresponding to the domain $\Omega \subset \mathbb{R}^{3}$. For any $\alpha \in \mathbb{R}$, the equation

$$
\alpha+\frac{\sqrt{-\xi}}{4 \pi}+h\left(\underline{x}_{0}, \underline{x}_{0}, \sqrt{-\xi}\right)=0, \quad \xi \in\left(-\infty, \lambda_{0}\right)
$$

admits a unique solution, denoted $\xi(\alpha)$, such that

$$
\lim _{\alpha \rightarrow-\infty} \xi(\alpha)=-\infty, \quad \xi\left(-h\left(\underline{x}_{0}, \underline{x}_{0}, 0\right)\right)=0,
$$

and

$$
\lim _{\alpha \rightarrow+\infty} \xi(\alpha)=\lambda_{0}
$$

Proof. In order to study solutions of (3.17), we need to find the dependence of $h\left(\underline{x}_{0}, \underline{x}_{0}, \sqrt{-\xi}\right)$ on the variable $\sqrt{-\xi}$. We start by considering the case $\xi \leq 0$. Setting $y=\sqrt{|\xi|}$, the equation (3.17) assumes the form

$$
\frac{y}{4 \pi}=-\alpha-h\left(\underline{x}_{0}, \underline{x}_{0}, y\right)=0, \quad y \geq 0
$$

and its solutions can be geometrically interpreted as the abscissas of the intersection points between the curves at the left and the right-hand side of (3.20). We will show that for any fixed choice of $\underline{x}_{0} \in \Omega, h\left(\underline{x}_{0}, \underline{x}_{0}, y\right)$ is a positive and strictly decreasing function of the variable $y$, such that

$$
\lim _{y \rightarrow+\infty} h\left(\underline{x}_{0}, \underline{x}_{0}, y\right)=0 .
$$

Let us consider the boundary value problem

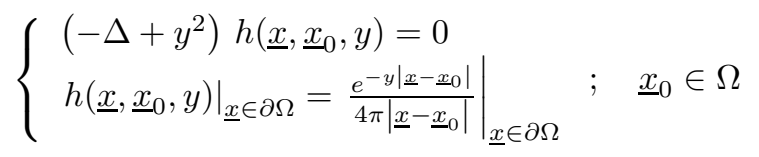

The solution of (3.22) is infinitely smooth in the open set $\Omega$, continuous and positive on its boundary. The strong maximum principle — cf. Ev98 — in this case allows us to claim that $h$ is strictly positive in $\Omega$ reaching its maximum on the boundary,

$$
0<h\left(\underline{x}, \underline{x}_{0}, y\right)<\sup _{\underline{x^{\prime}} \in \partial \Omega} \frac{e^{-y\left|\underline{x^{\prime}}-\underline{x}_{0}\right|}}{4 \pi\left|\underline{x^{\prime}}-\underline{x}_{0}\right|} \quad \text { for } \forall \underline{x} \in \Omega, y \geq 0 .
$$


Furthermore. the derivative $\partial_{y} h$ satisfies the equation

$$
\left\{\begin{array}{l}
\left(-\Delta+y^{2}\right) \partial_{y} h\left(\underline{x}, \underline{x}_{0}, y\right)=-2 y h\left(\underline{x}, \underline{x}_{0}, y\right) \\
\left.\partial_{y} h\left(\underline{x}, \underline{x}_{0}, y\right)\right|_{\underline{x} \in \partial \Omega}=-\left.\frac{e^{-y\left|\underline{x}-\underline{x}_{0}\right|}}{4 \pi}\right|_{\underline{x} \in \partial \Omega}
\end{array} ; \quad \underline{x}_{0} \in \Omega\right.
$$

the solution of which belongs to $C^{2}(\Omega) \cap C(\bar{\Omega})$ in view of the regularity of the source term and the boundary value. The maximum principle — see the version given in Br83, Thm IX.27] in this case implies

$$
\partial_{y} h\left(\underline{x}, \underline{x}_{0}, y\right)<0 \quad \text { for } \forall \underline{x} \in \Omega, y>0 .
$$

In particular, the solution of (3.24) for $y=0$ is $\partial_{y} h\left(\underline{x}, \underline{x}_{0}, 0\right)=-\frac{1}{4 \pi}$. This characterization of $h\left(\underline{x}_{0}, \underline{x}_{0}, y\right)$ allows us to claim that the equation (3.20) admits at least one solution for any $\alpha \in\left(-\infty,-h\left(\underline{x}_{0}, \underline{x}_{0}, 0\right)\right]$ and that the conditions (3.18) hold. Moreover, the second one of the relations (3.3) in Lemma 3.1 implies the monotonicity of the function $\alpha \mapsto y(\alpha)$ implicitly defined by (3.20); this grants the uniqueness of the solution.

Next we turn to (3.17) for $\xi \in\left(0, \lambda_{0}\right)$. In this case, setting $y:=\sqrt{\xi}$, the equation reads as

$$
\alpha+\frac{i y}{4 \pi}+h\left(\underline{x}_{0}, \underline{x}_{0}, i y\right)=0 .
$$

According to the second one of the relations (3.4), this is equivalent to

$$
\alpha+\operatorname{Re} h\left(\underline{x}_{0}, \underline{x}_{0}, i y\right)=0,
$$

where Re $h$ satisfies the boundary value problem

$$
\left\{\begin{array}{l}
\left(-\Delta-y^{2}\right) \operatorname{Re} h\left(\underline{x}, \underline{x}_{0}, i y\right)=0 \\
\left.\operatorname{Re} h\left(\underline{x}, \underline{x}_{0}, y\right)\right|_{\underline{x} \in \partial \Omega}=\left.\frac{\cos y\left|\underline{x}-\underline{x}_{0}\right|}{4 \pi\left|\underline{x}-\underline{x}_{0}\right|}\right|_{\underline{x} \in \partial \Omega} \quad ; \quad \underline{x}_{0} \in \Omega
\end{array}\right.
$$

It is worthwhile to notice that $h\left(\underline{x}, \underline{x}_{0}, y\right)$ is not defined for $y=\sqrt{\lambda_{0}}$. In particular, one can show that 2

$$
\lim _{|\varepsilon| \rightarrow 0}\left\|\varepsilon h\left(\cdot, \underline{x}_{0}, i \sqrt{\lambda_{0}-\varepsilon}\right)+\psi_{0}\left(\underline{x}_{0}\right) \psi_{0}(\cdot)\right\|_{L^{2}(U)}=0
$$

where $\psi_{0}$ is the principal eigenstate of the Dirichlet Laplacian and $U$ is any subset of $\Omega$. In view of the boundedness of $\psi_{0}$ and the arbitrariness of $U$, this relation also implies

$$
\lim _{y \rightarrow \sqrt{\lambda_{0}}-}\left|h\left(\underline{x}_{0}, \underline{x}_{0}, i y\right)\right|=+\infty
$$

Using this result together with the conditions (3.4) and (3.5) of Lemma 3.1 we conclude that $\operatorname{Re} h\left(\underline{x}_{0}, \underline{x}_{0}, i y\right)$ is a strictly decreasing function of $y \in\left(0, \sqrt{\lambda_{0}}\right)$ whose behavior for $y \rightarrow \sqrt{\lambda_{0}}$ is given by

$$
\lim _{y \rightarrow{\sqrt{\lambda_{0}}}^{-}} \operatorname{Re} h\left(\underline{x}_{0}, \underline{x}_{0}, i y\right)=-\infty
$$

Summing up this discussion, the equation (3.27) has a unique positive solution $y=y(\alpha)$ for any $\alpha \in\left(-h\left(\underline{x}_{0}, \underline{x}_{0}, 0\right),+\infty\right)$, and this solution asymptotically approaches the value $\lambda_{0}$ as $\alpha \rightarrow+\infty$; in combination with the first part this concludes the proof of the lemma.

\footnotetext{
${ }^{2}$ Relation (3.29) easily follows, e.g., from Lemma 2 in [BFM07.
} 
Next we deal with the eigenvalue equation in the two-dimensional case. Recall that the free Green's function related to this problem is the modified Bessel function $K_{0}$, which is strictly positive and convex in $\mathbb{R}^{+}$and admits the following representation AS72.

$$
K_{0}(x)=-\left\{\ln \frac{x}{2}+\gamma\right\} I_{0}(x)+\sum_{n=1}^{+\infty} c_{n} \frac{x^{2 n}}{(2 n !)^{2 n}}
$$

where $\gamma \approx 0.577$ is the Euler-Mascheroni constant, $c_{n}=\sum_{k=1}^{n} \frac{1}{k}$, and $I_{0}(x)$ is the other modified Bessel function given by the series

$$
I_{0}(x)=\sum_{n=0}^{+\infty} \frac{x^{2 n}}{(2 n !)^{2 n}}
$$

In the following proof we will make use of the asymptotic properties of $K_{0}$,

$$
\lim _{x \rightarrow 0^{+}} K_{0}(x)=+\infty, \quad \lim _{x \rightarrow+\infty} K_{0}(x)=0
$$

Lemma 3.3 Let $\lambda_{0}$ denote the first eigenvalue of $-\Delta_{\Omega}^{D}$ corresponding to the domain $\Omega \subset \mathbb{R}^{2}$. For any $\alpha \in \mathbb{R}$, the equation

$$
\alpha-\ln \sqrt{-\xi}-2 \pi h\left(\underline{x}_{0}, \underline{x}_{0}, \sqrt{-\xi}\right)=0, \quad \xi \in\left(-\infty, \lambda_{0}\right),
$$

admits an unique solution, denoted $\xi(\alpha)$, such that

$$
\lim _{\alpha \rightarrow+\infty} \xi(\alpha)=-\infty, \quad \xi\left(f\left(\underline{x}_{0}, \underline{x}_{0}, 0\right)\right)=0,
$$

and

$$
\lim _{\alpha \rightarrow-\infty} \xi(\alpha)=\lambda_{0}
$$

where $f\left(\underline{x}, \underline{x}_{0}, \sqrt{-\xi}\right)$ in the second one of the relations (3.36) is a $C^{+\infty}(\Omega) \cap C(\bar{\Omega})-$ regular function of both the spatial variables defined by

$$
f\left(\underline{x}, \underline{x}_{0}, \sqrt{-\xi}\right)=2 \pi h\left(\underline{x}, \underline{x}_{0}, \sqrt{-\xi}\right)+\ln \sqrt{-\xi} I_{0}\left(\sqrt{-\xi}\left|\underline{x}-\underline{x}_{0}\right|\right), \quad \xi<\lambda_{0} .
$$

Proof. The argument follows the same line as in Lemma 3.2, the main difference coming from the specific form of the Green functions in two dimensions. For $\xi \leq 0$ and $y:=\sqrt{|\xi|}$, equation (3.35) reads

$$
\alpha=\ln y+2 \pi h\left(\underline{x}_{0}, \underline{x}_{0}, y\right)
$$

where $h\left(\underline{x}, \underline{x}_{0}, \sqrt{y}\right)$, the solution of the boundary value problem

$$
\left\{\begin{array}{l}
\left(-\Delta+y^{2}\right) h\left(\underline{x}, \underline{x}_{0}, y\right)=0 \\
\left.h\left(\underline{x}, \underline{x}_{0}, y\right)\right|_{\underline{x} \in \partial \Omega}=\left.\frac{1}{2 \pi} K_{0}\left(y\left|\underline{x}-\underline{x}_{0}\right|\right)\right|_{\underline{x} \in \partial \Omega}
\end{array} ; \quad \underline{x}_{0} \in \Omega\right.
$$

is strictly positive in $\Omega$, as it follows from the maximum principle and the positivity of the boundary values. Consequently, the r.h.s. of (3.39) diverges as $y \rightarrow+\infty$. Moreover, from (3.3) we know that $\ln y+2 \pi h\left(\underline{x}_{0}, \underline{x}_{0}, y\right)$ is strictly increasing as a function of $y$ in the whole $\mathbb{R}^{+}$. In 
order to study the behaviour for $y \rightarrow 0$, we introduce the auxiliary function $f\left(\underline{x}, \underline{x}_{0}, y\right)$, defined in (3.38) which solves the boundary value problem

$$
\left\{\begin{array}{l}
\left(-\Delta+y^{2}\right) f\left(\underline{x}, \underline{x}_{0}, y\right)=0 \\
\left.h\left(\underline{x}, \underline{x}_{0}, y\right)\right|_{\underline{x} \in \partial \Omega}=\frac{1}{2 \pi} K_{0}\left(y\left|\underline{x}-\underline{x}_{0}\right|\right)+\left.\ln y I_{0}\left(\sqrt{-\xi}\left|\underline{x}-\underline{x}_{0}\right|\right)\right|_{\underline{x} \in \partial \Omega} \quad ; \quad \underline{x}_{0} \in \Omega
\end{array}\right.
$$

Using the definition (3.33), it is easy to verify that the quantity $\ln y+2 \pi h\left(\underline{x}_{0}, \underline{x}_{0}, y\right)$ coincides with the value of $f$ in $\underline{x}_{0}$, thus for $y \rightarrow 0^{+}$we have

$$
\lim _{y \rightarrow 0^{+}}\left(\ln y+2 \pi h\left(\underline{x}_{0}, \underline{x}_{0}, y\right)\right)=\lim _{y^{+} \rightarrow 0} f\left(\underline{x}_{0}, \underline{x}_{0}, y\right) .
$$

In this limit, the problem (3.41) simplifies to

$$
\left\{\begin{array}{l}
-\Delta f\left(\underline{x}, \underline{x}_{0}, 0\right)=0 \\
\left.h\left(\underline{x}, \underline{x}_{0}, 0\right)\right|_{\underline{x} \in \partial \Omega}=-\left.\frac{1}{2 \pi}\left\{\ln \frac{\left|\underline{x}-\underline{x}_{0}\right|}{2}+\gamma\right\}\right|_{\underline{x} \in \partial \Omega} \quad ; \quad \underline{x}_{0} \in \Omega
\end{array}\right.
$$

where the representation (3.32) has been considered; the regularity of the boundary condition in (3.42) implies $f\left(\underline{x}, \underline{x}_{0}, 0\right) \in C^{+\infty}(\Omega) \cap C(\bar{\Omega})$. So far we have shown that $\left(\ln y+2 \pi h\left(\underline{x}_{0}, \underline{x}_{0}, y\right)\right)$ is a strictly increasing function of $y \in \mathbb{R}^{+}$such that

$$
\lim _{y \rightarrow+\infty}\left(\ln y+2 \pi h\left(\underline{x}_{0}, \underline{x}_{0}, y\right)\right)=+\infty
$$

and

$$
\lim _{y \rightarrow 0^{+}}\left(\ln y+2 \pi h\left(\underline{x}_{0}, \underline{x}_{0}, y\right)\right)=f\left(\underline{x}_{0}, \underline{x}_{0}, 0\right) .
$$

From here we can conclude that the equation (3.35) admits an unique solution, $\xi(\alpha) \leq 0$, for any $\alpha \in\left[f\left(\underline{x}_{0}, \underline{x}_{0}, 0\right),+\infty\right)$, which satisfies the conditions (3.36).

In the case of a positive eigenvalue $\xi \in\left(0, \lambda_{0}\right)$, setting $y=\sqrt{\xi}$ we rewrite equation (3.35) as

$$
\alpha=\ln i y+2 \pi h\left(\underline{x}_{0}, \underline{x}_{0}, i y\right)
$$

which due to (3.4) is equivalent to

$$
\alpha=\ln y+2 \pi \operatorname{Re} h\left(\underline{x}_{0}, \underline{x}_{0}, i y\right) .
$$

As in the 3D case, we notice that the r.h.s of (3.46) is a strictly decreasing function, cf. (3.5), diverging as $y \rightarrow \sqrt{\lambda_{0}}$; the sought conclusion easily follows.

Since the spectrum of $H_{\alpha}$ is determined by the solutions of the equations (3.1), the above lemmata have the following implication which means that in a sense point interactions in dimension two and three can be always regarded as "attractive".

Corollary 3.4 For any real $\alpha$, the operator $H_{\alpha}$ defined by (2.8)-2.10) has a unique spectral point below the spectral threshold of $-\Delta_{\Omega}^{D}$.

\section{Dependence of the principal eigenvalue on the position of the interaction}

Now we pass to our main topic. We will characterize the behaviour of the principal eigenvalue of the point-interaction Hamiltonians $H_{\alpha}$ for a fixed bounded domain $\Omega \subset \mathbb{R}^{d}, d=2,3$, as the interaction site moves towards the boundary of $\Omega$. We will restrict our attention to domains having an interior reflection property w.r.t. a suitable hyperplane, in the following sense: 
Definition 4.1 Consider a hyperplane $P$ of dimension $d-1$ in $\mathbb{R}^{d}$ and denote by $S^{P}$ the mirror image of a set $S \subset \mathbb{R}^{d}$ w.r.t. $P$ provided $S \cap P=\varnothing$. The domain $\Omega$ is said to have the interior reflection property w.r.t. $P$ if $P \cap \Omega \neq \varnothing$ and there is an open connected component $\Omega_{s} \subset \Omega \backslash P$ such that $\Omega_{s}^{P}$ is a proper subset of $\Omega \backslash \bar{\Omega}_{s}$. We call $\Omega_{s}$ the smaller side of $\Omega$ and $P$ an interior reflection hyperplane.

To prove our main result, we need following auxiliary statement.

Lemma 4.2 Let $\mathcal{G}_{0}^{z}\left(\underline{x}, \underline{x}^{\prime}\right)$ be defined by (2.5)-(2.6) and $z \in \mathbb{R}$. For values of $z$ above $-\lambda_{0}$, the following implications hold,

$$
z \in[0,+\infty) \Longrightarrow \mathcal{G}_{0}^{z}\left(\underline{x}, \underline{x}^{\prime}\right)>0 \quad \text { in } \Omega
$$

and

$$
z \in\left(-\lambda_{0}, 0\right): \Longrightarrow: \operatorname{Re} \mathcal{G}_{0}^{z}\left(\underline{x}, \underline{x}^{\prime}\right)>0 \quad \text { in } \Omega .
$$

Proof. For $\Omega \subset \mathbb{R}^{3}$ and $z \in[0,+\infty)$, the Green function

$$
\mathcal{G}_{0}^{z}\left(\underline{x}, \underline{x}^{\prime}\right)=\frac{e^{-\sqrt{z}\left|\underline{x}-\underline{x}^{\prime}\right|}}{4 \pi\left|\underline{x}-\underline{x}^{\prime}\right|}-h\left(\underline{x}, \underline{x}^{\prime}, \sqrt{z}\right)
$$

is certainly positive in a small enough open neighbourhood $B_{\underline{x}^{\prime}}$ of the point $\underline{x}^{\prime}$ due to the boundedness of $h\left(\underline{x}, \underline{x}^{\prime}, \sqrt{z}\right)$. Moreover, it solves the boundary value problem

$$
\left\{\begin{array}{l}
(-\Delta+z) \mathcal{G}_{0}^{z}\left(\underline{x}, \underline{x}^{\prime}\right)=0 \quad \text { in } \Omega \backslash B_{\underline{x}^{\prime}} \\
\left.\mathcal{G}_{0}^{z}\right|_{\partial \Omega}=0, \quad ;\left.\quad \mathcal{G}_{0}\right|_{\partial B_{\underline{x}^{\prime}}}>0
\end{array} \quad \underline{x}^{\prime} \in \Omega\right.
$$

It follows from the maximum principle that $\mathcal{G}_{0}^{z}$ is strictly positive in the whole $\Omega$.

To prove the other implication in the $3 \mathrm{D}$ case, notice that for $z \in\left(-\lambda_{0}, 0\right)$ we have

$$
\operatorname{Re} \mathcal{G}_{0}^{z}\left(\underline{x}, \underline{x}^{\prime}\right)=\frac{\cos \sqrt{|z|}\left|\underline{x}-\underline{x}^{\prime}\right|}{4 \pi\left|\underline{x}-\underline{x}^{\prime}\right|}-\operatorname{Re} h\left(\underline{x}, \underline{x}^{\prime}, i \sqrt{|z|}\right) .
$$

Once more we can find a suitable open neighbourhood of the point $\underline{x}^{\prime}$, which we call $B_{\underline{x}^{\prime}}$, where this function is positive. In $\Omega \backslash B_{\underline{x}^{\prime}} \operatorname{Re} \mathcal{G}_{0}^{z}\left(\underline{x}, \underline{x}^{\prime}\right)$ solves the boundary value problem

$$
\left\{\begin{array}{l}
(-\Delta+z) \operatorname{Re} \mathcal{G}_{0}^{z}\left(\underline{x}, \underline{x}^{\prime}\right)=0 \quad \text { in } \Omega \backslash B_{\underline{x}^{\prime}} \\
\left.\mathcal{G}_{0}\right|_{\partial \Omega}=0, \quad ;\left.\quad \mathcal{G}_{0}\right|_{\partial B_{\underline{x}^{\prime}}}>0
\end{array}\right.
$$

Under the condition $z>-\lambda_{0}$ we can still apply the maximum principle obtaining in this way $\operatorname{Re} \mathcal{G}_{0}^{z}\left(\underline{x}, \underline{x}^{\prime}\right)>0$ in $\Omega$. Finally, in the $2 \mathrm{D}$ case the proof follows the same line with the replacement (4.3) and (4.5) by the corresponding 2D Green's function

$$
\mathcal{G}_{0}^{z}\left(\underline{x}, \underline{x}^{\prime}\right)=K_{0}\left(\sqrt{z}\left|\underline{x}-\underline{x}^{\prime}\right|\right)-h\left(\underline{x}, \underline{x}^{\prime}, \sqrt{z}\right)
$$

and taking into account the asymptotic properties of $K_{0}(x)$ as $x \rightarrow 0$.

Now we are in position to prove our main result. The next theorem shows that, under the interior reflection conditions imposed on the domain $\Omega$, the principal eigenvalue of $H_{\alpha}$ increases as the interaction site moves towards the boundary of the smaller side $\Omega_{s}$ of $\Omega$.

Theorem 4.3 Let $P$ be an interior reflection hyperplane for the domain $\Omega$ and denote by $\underline{n}$ the normal vector to $P$ pointing towards $\Omega_{s}$. Assume that $\underline{x}_{0} \in \Omega \cap\left(\partial \Omega_{s} \cap P\right)$; then the principal eigenvalue $\xi$ of the point-interaction $H_{\alpha}$ with the perturbation placed at $\underline{x}_{0}$ satisfies the condition

$$
\underline{n} \cdot \nabla_{\underline{x}_{0}} \xi>0 \text {. }
$$


Proof. Consider first the $3 \mathrm{D}$ case. To analyze the dependence of $\xi$ on the interaction position $\underline{x}_{0}$, we have to distinguish between the negative and positive spectral points. If $\alpha \in$ $\left(-\infty,-h\left(\underline{x}_{0}, \underline{x}_{0}, 0\right)\right]$, then $\xi=\xi\left(\underline{x}_{0}\right)$ is by Lemma 3.2 a negative solution of (3.20). Replacing $\sqrt{|\xi|}$ with $y\left(\underline{x}_{0}\right)$ and taking the gradient w.r.t. $\underline{x}_{0}$ in $(3.20)$ we find

$$
\nabla_{\underline{x}_{0}} y\left(\underline{x}_{0}\right)\left(\frac{1}{4 \pi}+\partial_{y} h\right)=-\nabla_{\underline{x}_{0}} h\left(\underline{x}_{0}, \underline{x}_{0}, y\right) \text {. }
$$

Next we consider the term $\nabla_{\underline{x}_{0}} h\left(\underline{x}_{0}, \underline{x}_{0}, y\right)$ at the r.h.s. of the last equation; under our interior reflection assumptions we will show that this vector is oriented towards the smaller side of $\Omega$. To this aim we notice that, in view of the relations (2.2)-(2.4), $h\left(\underline{x}, \underline{x}^{\prime}, y\right)$ can be written as

$$
h\left(\underline{x}, \underline{x}^{\prime}, y\right)=\frac{e^{-y\left|\underline{x}-\underline{x}^{\prime}\right|}}{4 \pi\left|\underline{x}-\underline{x}^{\prime}\right|}-\sum_{\substack{n \in \mathbb{N}_{0} \\ k \leq N_{n}}} \frac{\psi_{n, k}\left(\underline{x}^{\prime}\right) \psi_{n, k}(\underline{x})}{\lambda_{n}+y^{2}}
$$

for any $\underline{x} \neq \underline{x}^{\prime}$. From the symmetry of this expression and the regularity $h\left(\underline{x}, \underline{x}^{\prime}, y\right)$ it follows that

$$
\left.\nabla_{\underline{x}^{\prime}} h\left(\underline{x}, \underline{x}^{\prime}, y\right)\right|_{\underline{x}=\underline{x}^{\prime}=\underline{x}_{0}}=\left.\nabla_{\underline{x}} h\left(\underline{x}, \underline{x}^{\prime}, y\right)\right|_{\underline{x}=\underline{x}^{\prime}=\underline{x}_{0}}
$$

and

$$
\nabla_{\underline{x}_{0}} h\left(\underline{x}_{0}, \underline{x}_{0}, y\right)=\left.2 \nabla_{\underline{x}} h\left(\underline{x}, \underline{x}^{\prime}, y\right)\right|_{\underline{x}_{=}=\underline{x}^{\prime}=\underline{x}_{0}} .
$$

To analyze the orientation of this vector, we introduce the function $u$ defined on the smaller part of $\Omega$ by

$$
u\left(\underline{x}, \underline{x}_{0}, y\right)=h\left(\underline{x}, \underline{x}_{0}, y\right)-h\left(\underline{x}^{P}, \underline{x}_{0}, y\right), \quad \underline{x} \in \Omega_{s},
$$

where $\underline{x}^{P}$ denotes the mirror image of $\underline{x} \in \Omega_{s}$ through the plane $P$. The following equation holds

$$
\left\{\begin{array}{l}
\left(-\Delta+y^{2}\right) u=0 \quad \text { in } \Omega_{s} \\
\left.u\right|_{P \cap \Omega}=0,\left.\quad u\right|_{\partial \Omega_{s} \cap \partial \Omega}=\frac{e^{-y\left|\underline{x}_{0} \underline{x}_{0}\right|}}{4 \pi\left|\underline{x}-\underline{x}_{0}\right|}-\left.h\left(\underline{x}^{P}, \underline{x}_{0}, y\right)\right|_{\underline{x}_{x} \in \partial \Omega s \cap \partial \Omega}
\end{array} \quad ; \quad \underline{x}_{0} \in \Omega \cap P\right.
$$

It is worthwhile to notice that the boundary value on $\partial \Omega_{s} \cap \partial \Omega$ can be identified with the value of $\mathcal{G}_{0}^{y^{2}}\left(\underline{x}, \underline{x}_{0}\right)$ on the set $(\partial \Omega s \cap \partial \Omega)^{P}$, indeed we have

$$
\left.\mathcal{G}_{0}^{y^{2}}\left(\underline{x}, \underline{x}_{0}\right)\right|_{(\partial \Omega s \cap \partial \Omega)^{P}}=\frac{e^{-y\left|\underline{x}^{-} \underline{x}_{0}\right|}}{4 \pi\left|\underline{x}-\underline{x}_{0}\right|}-\left.h\left(\underline{x}, \underline{x}_{0}, y\right)\right|_{(\partial \Omega s \cap \partial \Omega)^{P}}=\frac{e^{-y\left|\underline{x}-\underline{x}_{0}\right|}}{4 \pi\left|\underline{x}-\underline{x}_{0}\right|}-\left.h\left(\underline{x}^{P}, \underline{x}_{0}, y\right)\right|_{\partial \Omega s \cap \partial \Omega}
$$

Then it follows from (4.1) that $u$ is positive on $\partial \Omega s \cap \partial \Omega$ and by the maximum principle, $u>0$ holds in $\Omega_{s}$. In particular, $u$ reaches its minimum on the points of the open surface $P \cap \Omega$; the Hopf boundary-point lemma in this case implies

$$
\underline{n} \cdot \nabla_{\underline{x}} u>0 \quad \text { for } \forall \underline{x} \in P \cap \Omega .
$$

Due to the definition (4.13), in combination with the relation

$$
\underline{n} \cdot \nabla_{\underline{x}} h\left(\underline{x}^{P}, \underline{x}_{0}, y\right)=-\underline{n} \cdot \nabla_{\underline{x}^{P}} h\left(\underline{x}^{P}, \underline{x}_{0}, y\right) \quad \text { in } \Omega_{s} \cup(P \cap \Omega),
$$

the last inequality also implies

$$
2 \underline{n} \cdot \nabla_{\underline{x}} h\left(\underline{x}, \underline{x}_{0}, y\right)>0 \quad \text { for } \forall \underline{x} \in P \cap \Omega .
$$


Substituting (4.12) and (4.18) into the r.h.s. of (4.9) and taking the projection in the direction of the vector $\underline{n}$ we get

$$
\underline{n} \cdot \nabla_{\underline{x}_{0}} y\left(\underline{x}_{0}\right)\left(\frac{1}{4 \pi}+\partial_{y} h\right)=-\underline{n} \cdot \nabla_{\underline{x}_{0}} h\left(\underline{x}_{0}, \underline{x}_{0}, y\right)<0 .
$$

The term $\nabla_{\underline{x}_{0}} y\left(\underline{x}_{0}\right)$ at the l.h.s. of (4.19) is related to $\nabla_{\underline{x}_{0}} \xi$ by

$$
\nabla_{\underline{x}_{0}} y=-\frac{1}{y} \nabla_{\underline{x}_{0}} \xi
$$

from which it follows that

$$
\frac{1}{y}\left(\frac{1}{4 \pi}+\partial_{y} h\right) \nabla_{\underline{x}_{0}} \xi>0
$$

The sought inequality (4.8) follows easily from (4.21) taking into account the condition (3.3).

In the opposite case, $\alpha>-h\left(\underline{x}_{0}, \underline{x}_{0}, 0\right)$, the first spectral point of $H_{\alpha}$ is a strictly positive solution of the equation

$$
\alpha+\operatorname{Re} h\left(\underline{x}_{0}, \underline{x}_{0}, i \sqrt{\xi}\right)=0
$$

with $\xi<\lambda_{0}-$ cf. (3.27) in Lemma 3.2 Replacing $\sqrt{\xi}$ with $y\left(\underline{x}_{0}\right)$ and taking the gradient w.r.t. $\underline{x}_{0}$ in (4.22), we get

$$
\nabla_{\underline{x}_{0}} y \partial_{y} \operatorname{Re} h\left(\underline{x}_{0}, \underline{x}_{0}, i y\right)=-\nabla_{\underline{x}_{0}} \operatorname{Re} h\left(\underline{x}_{0}, \underline{x}_{0}, i y\right) .
$$

In order to check the orientation of the vector at the r.h.s of this expression, we notice again that due to the symmetry of the function

$$
\operatorname{Re} h\left(\underline{x}, \underline{x}^{\prime}, i y\right)=\frac{\cos y\left|\underline{x}-\underline{x}^{\prime}\right|}{4 \pi\left|\underline{x}-\underline{x}^{\prime}\right|}-\sum_{\substack{n \in \mathbb{N}_{0} \\ k \leq N_{n}}} \frac{\psi_{n, k}\left(\underline{x}^{\prime}\right) \psi_{n, k}(\underline{x})}{\lambda_{n}-y^{2}}
$$

the gradient $\nabla_{\underline{x}_{0}} \operatorname{Re} h\left(\underline{x}_{0}, \underline{x}_{0}, i y\right)$ can be expressed as

$$
\nabla_{\underline{x}_{0}} \operatorname{Re} h\left(\underline{x}_{0}, \underline{x}_{0}, i y\right)=\left.2 \nabla_{\underline{x}} \operatorname{Re} h\left(\underline{x}, \underline{x}^{\prime}, y\right)\right|_{\underline{x}^{\prime}=\underline{x}^{\prime}=\underline{x}_{0}} .
$$

Then we follow the line of the first part of the proof introducing the function $u$,

$$
u\left(\underline{x}, \underline{x}_{0}, y\right)=\operatorname{Re} h\left(\underline{x}, \underline{x}_{0}, i y\right)-\operatorname{Re} h\left(\underline{x}^{P}, \underline{x}_{0}, i y\right), \quad \underline{x} \in \Omega_{s} .
$$

Proceeding as before and taking into account the implication (4.2) we find easily

$$
\underline{n} \cdot \nabla_{\underline{x}} u>0
$$

and

$$
2 \underline{n} \cdot \nabla_{\underline{x}} \operatorname{Re} h\left(\underline{x}, \underline{x}_{0}, y\right)>0 \quad \text { for } \forall \underline{x} \in P \cap \Omega ;
$$

substituting (4.25) and (4.28) into the r.h.s. of (4.23), we conclude that

$$
\underline{n} \cdot \nabla_{\underline{x}_{0}} y \partial_{y} \operatorname{Re} h\left(\underline{x}_{0}, \underline{x}_{0}, i y\right)<0
$$

The claim (4.8) is then obtained from (4.29) by taking into account the relation

$$
\nabla_{\underline{x}_{0}} y=\frac{1}{y} \nabla_{\underline{x}_{0}} \xi
$$

and the inequality (3.5) from Lemma 3.1 This concludes the argument in the three-dimensional case; the two-dimensional one can be dealt with in the same way, step by step. 


\section{Optimization of $\xi\left(\underline{x}_{0}\right)$}

By Theorem 4.3 the spectral threshold of the operator $H_{\alpha}$ increases as the interaction position $\underline{x}_{0}$ moves towards the boundary of the domain $\Omega$. This result provides us with some insights on how to place the point-interaction centre to minimize the principal eigenvalue of the Hamiltonian $H_{\alpha}$. For the sake of simplicity, we begin with the case of a convex $\Omega$. Let $\Pi$ be the set of all the hyperplanes $P$ of interior reflection for $\Omega$; we denote by $\Omega_{s, P}$ the smaller part related to $P \in \Pi$, provided it exists, and by $\Sigma$ the union

$$
\Sigma=\bigcup_{P \in \Pi} \Omega_{s, P}
$$

The following claim is a straightforward consequence of the Theorem 4.3 .

Corollary 5.1 Let $\Omega$ be an open convex domain in $\mathbb{R}^{d}, d=2,3$, and let $H_{\alpha}$ be a point-interaction operator in $\Omega$ with the perturbation placed at $\underline{x}_{0}$. The principal eigenvalue of $H_{\alpha}$, considered as a function of the interaction centre, takes its minimum value when $\underline{x}_{0}$ belongs to the open set $\Omega \backslash \Sigma$.

Proof. Notice first that the continuity of $h\left(\underline{x}, \underline{x}_{0}, y\right)$ implies the continuity of solutions of the eigenvalue equations (3.1), thus the principal eigenvalue $\xi\left(\underline{x}_{0}\right)$ has at least one minimum point $\underline{x}_{0}^{m} \in \bar{\Omega}$. We use reduction ad absurdum: assume $\underline{x}_{0}^{m} \in \Omega_{s, P}$ for a suitable hyperplane $P \in \Pi$. Due to the convexity of the domain, it exists another hyperplane $P^{\prime} \in \Pi$ parallel to $P$ and such that

$$
\underline{x}_{0}^{m} \in \partial \Omega_{s, P^{\prime}} \cap P^{\prime},
$$

however, under this assumption Theorem 4.3 implies the inequality

$$
\underline{n} \cdot \nabla_{\underline{x}_{0}} \xi>0
$$

from which a contradiction follows easily.

In the case of highly symmetric domains such as the interior of a circle or an ellipse in the plane, and similarly a ball and an interior of an ellipsoid in three dimensions, it is easy to identify the set $\Sigma$ with the center of such a domain. More generally, the convexity of $\Omega$ ensures the validity of the interior reflection property needed in Theorem 4.3 with respect to some hyperplane passing through a point sufficiently close to the boundary. In this situation the above result can be used to localize the optimal position of $\underline{x}_{0}$ in a 'central' subset of the domain $\Omega$.

A slight generalization of the above argument lead us to an analogous rule to localize the minimum points of the principal eigenvalue for a point interaction within non-convex domains. Let $P \in \Pi$ and consider the one parameter family of hyperplanes $P_{t}$,

$$
\left\{\begin{array}{c}
P_{t}=\{\underline{x}+\underline{n} t: \underline{x} \in P, t \in[0, T]\} \\
T=\max \left\{t \in \mathbb{R}^{+}: P_{t} \cap \Omega \neq \varnothing\right\}
\end{array}\right.
$$

where $\underline{n}$ denotes the unit normal to $P$ directed towards the smaller part $\Omega_{s}$ of $\Omega$. We denote as $\Pi^{\prime}$ the subset formed by all those hyperplanes $P \in \Pi$ such that

$$
P_{t} \in \Pi \quad \text { for } \forall t \in[0, T] .
$$

It is important to notice that in the non-convex case, to any hyperplane of interior reflection there may correspond more than one smaller part. Next we denote by $\Theta_{s, P}$ the union of all the smaller parts related to $P$, and by $\Sigma^{\prime}$ the set

$$
\Sigma^{\prime}=\bigcup_{P \in \Pi^{\prime}} \Theta_{s, P}
$$


Corollary 5.2 Assume that $\Omega$ is an open domain in $\mathbb{R}^{d}, d=2,3$, and $H_{\alpha}$ is a point-interaction operator in $\Omega$ with the perturbation placed at $\underline{x}_{0}$. The principal eigenvalue of $H_{\alpha}$, regarded as a function of $\underline{x}_{0}$, takes its minimum value when $\underline{x}_{0}$ belongs to the open set $\Omega \backslash \Sigma^{\prime}$.

Proof. The argument is an easy modification of the proof of Corollary 5.1 .

Non-convex domains with a reasonably simple boundary such as, for instance, the union of two intersecting disks or a dog-bone profile in two dimensions, can be easily analyzed using Corollary 5.2. It is also worthwhile to stress that the results of this section do not depend on the parameter $\alpha$, hence the optimal placement of the point interaction with respect to the minimum of the principal eigenvalues can be the same irrespective of the interaction "strength".

Let us finally comment on he relation to the work [HKK01] mentioned in the introduction. We have said that for a hard-wall obstacle the principal eigenvalue decreases as it moves towards the boundary. The difference of the two effects can be traced back to the different boundary conditions which characterize the operator domains in the two cases. While the hard obstacle is characterized by Dirichlet boundary condition, the point-interaction operator $H_{\alpha}$ considered here can be obtained as the norm-resolvent limit of a family of sphere interactions Hamiltonians $H_{\alpha}(r)$ with the boundary condition of a mixed type as the radius $r \rightarrow 0$. In the three-dimensional case, for instance, the operator $H_{\alpha}(r)$ is explicitly given by

$$
\left\{\begin{array}{l}
H_{\alpha}(r)=-\Delta \quad \text { on } \Omega \backslash S_{r} \\
D\left(H_{\alpha}(r)\right)=\left\{\psi \in H^{1}\left(\mathbb{R}^{3}\right) \mid\left(\partial_{n} \psi\right)_{+}-\left(\partial_{n} \psi\right)_{-}=\frac{1}{4 \pi \alpha r^{2}+r} \psi\right\}
\end{array}\right.
$$

where $S_{r}$ denotes the sphere of radius $r$ centered at $\underline{x}_{0}$ and $\left(\partial_{n} \psi\right)_{+}-\left(\partial_{n} \psi\right)_{-}$is the jump of the normal derivative of $\psi$ on the interaction surface FT93, Sh03. Another insight into the difference of the two situations can be obtained from EŠ

\section{Acknowledgments}

We wish to thank Rodolfo Figari and Francesco Chiacchio for their useful remarks. This research was partially supported by GAAS and MEYS of the Czech Republic under projects A100480501 and LC06002.

\section{References}

[AS72] M. Abramowitz and I.A. Stegun, ed.: Handbook of Mathematical functions, Dover, New York 1972.

[AG63] N.I. Akhiezer, I.M. Glazman. Theory of Linear Operators in Hilbert Space, Vol II, Frederick Ungar Publishing Co., New York 1963.

[AGHH05] S. Albeverio, F. Gesztesy, R Högh-Krohn and H. Holden: Solvable Models in Quantum Mechanics, 2nd edition, with an appendix by P. Exner, AMS, Providence, R.I, 2005.

[AB92a] M.S. Ashbaugh, R.D. Benguria: A sharp bound for the ratio of the first two eigenvalues of Dirichlet Laplacians and extensions, Ann. Math. 135 (1992), 601-628.

[AB92b] M.S. Ashbaugh, R.D. Benguria: A second proof of the Payne-Pólya-Weinberger conjecture, Commun.Math.Phys. 147 (1992), 181-190. 
[BFM07] Ph. Blanchard, R. Figari, A. Mantile: Point interaction Hamiltonians in bounded domains, J. Math. Phys. 48 (2007), 082108

[Br83] H. Brezis. Analyse fonctionelle, Masson, Paris 1983.

[Ev98] L.C. Evans. Partial Differential Equations, AMS, Providence, R.I., 1998.

[EGŠT96] P. Exner, R. Gawlista, P. Šeba, M. Tater: Point interactions in a strip, Ann. Phys. 252 (1996), 133-179.

[EN02] P. Exner, K. Němcová: Quantum mechanics of layers with a finite number of point perturbations, J.Math.Phys. 43 (2002), 1152-1184.

[EŠ96] P. Exner, P. Šeba: Point interactions in dimension two and three as models of small scatterers, Phys. Lett. A222 (1996), 1-4.

[Fa23] G. Faber: Beweiss das unter allen homogenen Membranen von Gleicher Fläche und gleicher Spannung die kreisförmige den Tiefsten Grundton gibt, Sitzungber. der math.-phys. Klasse der Bayerische Akad. der Wiss. zu München (1923), 169172.

[FT93] R. Figari, A. Teta: A boundary value problem of mixed type on perforated domains, Asymptotic Analysis 6 (1993), 271-284.

[HKK01] E.M. Harrell, P. Kröger, K. Kurata: On the placement of an obstacle or a well so as to optimize the fundamental eigenvalue, SIAM J. Math. Anal 33 (2001), 240-259.

[Kr25] E. Krahn: Über eine von Rayleigh formulierte minimal Eigenschaft des Kreises, Ann. Math. 94 (1925), 97-100.

[PPW55] L.E. Payne, G. Pólya, H.F. Weinberger: Sur le quotient de deux fréquences propres consecutives, Comp. Rend. 241 (1955), 917-919.

[RS75] M. Reed, B. Simon. Methods of Modern Mathematical Physics, II. Fourier Analysis, Self-Adjointness, Academic Press, New York 1975.

[RS78] M. Reed, B. Simon. Methods of Modern Mathematical Physics, IV. Analysis of Operators, Academic Press, New York 1978.

[Sh03] Shin-ichi Shimada: Resolvent convergence of sphere interactions to point interactions, J. Math. Phys. 44 (2003), 990-1005. 